\title{
Experiencia en el manejo de feocromocitoma en los últimos 10 años: serie de casos
}

\author{
Erika P. Navarro1, María Camila Osejo², Luz Ángela Casas ${ }^{3}$, Luis Guillermo Arango \\ Guillermo Guzmán ${ }^{3}$
}

\author{
${ }^{1}$ Residente de Medicina Interna, Universidad CES, Fundación Valle \\ del Lili, Cali, Colombia \\ ${ }^{2}$ Residente de Medicina Interna, Universidad ICESI, Fundación \\ Valle del Lili, Cali, Colombia \\ ${ }^{3}$ Unidad de Endocrinología, Fundación Valle del Lili, Cali, \\ Colombia
}

Correspondencia: Guillermo E Guzmán. Cra. 98 18-49, Cali, Colombia, Servicio de Endocrinología. Fundación Valle del Lili Correo electrónico: Email: guillermoeguzman@gmail.com Conflictos de interés: Los autores declaran que no existen conflictos de interés.

Fecha de recepción: 18/04/2016

Fecha de aceptación: 30/06/2016

\section{Resumen}

$\mathrm{E}$ feocromocitoma es un tumor productor de catecolaminas. Se describe la experiencia en el diagnóstico, manejo y seguimiento de pacientes con este diagnóstico en una institución de alta complejidad en Cali, Colombia, en los últimos 10 años. Se encontraron 11 casos con diagnóstico de feocromocitoma llevados a cirugía, dos de estos extraadrenal y uno maligno. Fueron asintomáticos 18\%, 81\% presentaron hipertensión arterial. La evolución posquirúrgica de los pacientes fue favorable, $33 \%$ persistieron con hipertensión arterial (HTA) luego de la resección del tumor, requiriendo menor dosis de antihipertensivos. El único caso de malignidad tuvo una respuesta no satisfactoria. A pesar de ser un diagnóstico infrecuente, su abordaje es importante debido a la morbilidad que representa. Las características de nuestra población son similares a lo reportado en la literatura, con baja frecuencia de complicaciones y adecuada respuesta al tratamiento.

Palabras claves: feocromocitoma, paranganglioma, tumores adrenales.

\section{Abstract}

Pheochromocytoma is a catecholamine-producing tumor. We describe the experience in the diagnosis, management and monitoring of patients with pheochromocytoma in a highly complex institution in Cali, Colombia, in the last 10 years. We identified
11 cases with diagnosis of pheochromocytoma, who were taken to surgery, being two of these extraadrenal and one of these malignant. $18 \%$ were asymptomatic, $81 \%$ had hypertension. The postoperative course of patients was favorable, 33\% persisted hypertensive after tumor resection, although less doses of blood pressure medication were required. The only case of malignancy had an unsatisfactory response. Despite being a rare diagnosis, the approach is important because morbidity in our population is similar to those reported in the literature, with a low frequency of complications and adequate response to treatment.

\section{Introducción}

Los feocromocitomas son raros tumores productores de catecolaminas (adrenalina, noradrenalina y ocasionalmente dopamina) que derivan de las células cromafines y se asientan habitualmente en la médula suprarrenal y, con menor frecuencia, en los paraganglios simpáticos, denominándose entonces paragangliomas funcionantes o feocromocitomas extra-adrenales $^{(1)}$. Tienen una prevalencia de un caso por cada 4.500 personas y una incidencia 3-8 casos por millón por año ${ }^{(2)}$. Las manifestaciones clínicas y bioquímicas son, en su mayor parte, el resultado del exceso de catecolaminas producido por el tumor. Pese a su escasa frecuencia, representa $0,05-0,1 \%{ }^{(2)}$ de los pacientes con cualquier causa de hipertensión arterial sostenida; sin embargo, su importancia radica en que representan una de las causas de HTA curable y en que la falta de un diagnóstico precoz conlleva un alto riesgo de morbimortalidad.

Hasta la fecha, los reportes de la experiencia en el manejo de feocromocitoma en Colombia son escasos, con una serie de casos publicada por D’Achiardi y colaboradores hace más de 30 años $^{(3)}$. A continuación se presenta una serie de 11 casos de pacientes con diagnóstico de feocromocitoma y paranganglioma confirmados por patología, evaluados y manejados en una institución de alta complejidad del suroccidente colombiano durante el periodo de 2006 a 2016.

\section{Materiales y métodos}

Esta es una serie de casos de pacientes con feocromocitoma que incluyó pacientes con diagnóstico de feocromocitoma 
confirmado por patología durante el periodo comprendido entre 2006 y 2016 (a la fecha) en la Fundación Clínica Valle del Lili. Se hizo una revisión de las bases de datos de patología de pacientes con diagnóstico de feocromocitoma durante el periodo de estudio. Las variables fueron edad, género, tamaño de la lesión, localización, descripción patológica, necesidad de manejo con alfa y betabloqueadores, requerimiento de vasodilatador, episodios de crisis hipertensiva antes o durante el procedimiento, hipotensión durante y posterior al procedimiento, requerimiento de medicamento vasoactivo, presencia de metástasis y correlación del diagnóstico patológico con el perfil bioquímico, síntomas y evolución en términos de persistencia de HTA y sobrevida durante el seguimiento.

Para el proceso de recolección de la información se diseñó un formato de recolección de datos de las variables descritas, los datos fueron obtenidos de las historias clínicas sistematizadas de la institución de nivel IV donde se llevó a cabo el estudio.

Se describieron las variables sociodemográficas, clínicas, bioquímicas e histopatológicas de los pacientes que se analizaron en el estudio a través de las medidas de tendencia central correspondientes y se determinó la proporción de cada categoría.

\section{Resultados}

Durante el periodo entre 2006 y 2016 se encontraron 11 pacientes con diagnóstico de feocromocitoma confirmado por patología, dentro de los cuales $45 \%(n=5)$ eran hombres y $55 \%(n=6)$ mujeres, con mediana de edad de 41 años. El 100\% $(\mathrm{n}=11)$ de los pacientes presentaban lesiones unilaterales, $90,9 \%(n=9)$ adrenales y $18 \%(n=2)$ extraadrenales. Se documentó un caso con tumor compuesto (ganglioneuroma y feocromocitoma), una paciente con paraganglioma extraadrenal y un caso de feocromocitoma maligno extradrenal originado en el proceso uncinado del páncreas con metástasis a sistema nervioso central y hueso que recibió manejo quirúrgico y con quimioterapia sin respuesta. Ninguno de los casos presentó lesión tumoral bilateral. La mediana del tamaño de las lesiones fue de 4,7 cm (tabla 1). En cuanto al perfil bioquímico de los pacientes, uno tenía metanefrinas totales calculadas en plasma, que eran positivas con un valor $1.865 \mathrm{pg} / \mathrm{ml}$ (VR 0,05-0,16 $\mathrm{pg} / \mathrm{ml}), 45 \%(\mathrm{n}=5)$ de los pacientes tenían medición por colorimetría de ácido vanil mandélico en orina de 24 horas, con un resultado promedio de $545 \mathrm{mg} / 24 \mathrm{~h}$ (VR 2-14 mg en $24 \mathrm{~h}$ ). El 36\% (n=4) de los pacientes tenían metanefrinas totales en orina de 24 horas medidas por cromatografía positivas con un promedio de $6.411 \mathrm{mcg} / 24 \mathrm{~h}$ (VR < $900 \mathrm{mcg} / 24 \mathrm{~h}$ ) (tabla 2).

La presentación clínica más común fue la hipertensión arterial, taquicardia, palpitaciones, sudoración, dolor abdominal y manifestaciones clínicas menos usuales como hematuria y miopatía. El 18\% (n=2) fueron totalmente asintomáticos (tabla 3).

El 55\% (n=6) de los pacientes se sometieron a resección tumoral vía laparoscópica, incluyendo un tumor de 7,0 cm. El
Tabla 1. Características de los pacientes diagnosticados con feocromocitoma 2006-2016

\begin{tabular}{|c|c|}
\hline Característica & $\begin{array}{c}\text { Pacientes } \\
\% \text { n (11) }\end{array}$ \\
\hline Edad (mediana) & 41 años \\
\hline \multicolumn{2}{|l|}{ Género } \\
\hline Masculino & $45 \%(5)$ \\
\hline Femenino & $55 \%(6)$ \\
\hline \multicolumn{2}{|l|}{ Localización del tumor } \\
\hline Adrenal & $90,9 \%(10)$ \\
\hline Extraadrenal & $18 \%(2)$ \\
\hline Tumor unilateral & $100 \%(11)$ \\
\hline Tamaño de la lesión (cm) & $4,7 \mathrm{~cm}$ \\
\hline Patología maligna & $9,09 \%(1)$ \\
\hline \multicolumn{2}{|l|}{ Uso de alfa bloqueadores prequirúrgicos } \\
\hline Prazosin & $55 \%(6)$ \\
\hline Uso de betabloqueadores prequirúrgicos & $63 \%(7)$ \\
\hline Metoprolol & $71 \%(5)$ \\
\hline Carvedilol & $28,5 \%(2)$ \\
\hline $\begin{array}{l}\text { Uso de terapia alfa y betabloqueante } \\
\text { combinada prequirúrgica }\end{array}$ & $45 \%(5)$ \\
\hline $\begin{array}{l}\text { Tratamiento farmacológico desconocido } \\
\text { prequirúrgico }\end{array}$ & $27 \%(3)$ \\
\hline Crisis hipertensiva & $27 \%(3)$ \\
\hline Uso de nitroprusiato & $18 \%(2)$ \\
\hline Hipotensión posquirúrgica & $55 \%(6)$ \\
\hline Soporte vasopresor (norepinefrina) & $45 \%(5)$ \\
\hline \multicolumn{2}{|l|}{ Intervención quirúrgica } \\
\hline Laparotomía & $36 \%(4)$ \\
\hline Laparoscopia & $54 \%(6)$ \\
\hline Sin información & $9 \%(1)$ \\
\hline Complicaciones posquirúrgicas & $9 \%(1)$ \\
\hline Mortalidad & $0 \%(0)$ \\
\hline
\end{tabular}

Datos poblacionales de los pacientes identificados en el estudio con respecto a las variables estudiadas.

$45 \%(n=5)$ recibieron alfa bloqueador, encontrando que $27 \%$ $(n=3)$ presentaron hipertensión en el momento de la manipulación de tumor, requiriendo soporte vasodilatador (nitroprusiato) el 18\% ( $n=2)$. El 45\% ( $n=5)$ de los pacientes requirieron soporte vasopresor con norepinefrina por un tiempo no mayor a 48 horas; el resto toleraron y no presentaron cambios hemodinámicos importantes.

Del total de pacientes, $9(81 \%)$ tenían diagnóstico previo de hipertensión arterial y posterior a la cirugía sólo 3 de los 9 (33\%) persistieron con hipertensión arterial, aunque lograron 
Tabla 2. Comportamiento del perfil bioquímico de los casos

\begin{tabular}{|c|c|c|c|c|c|}
\hline Catecolaminas & Técnica de laboratorio & Media & Mediana & $\begin{array}{l}\text { Valor } \\
\text { mínimo }\end{array}$ & $\begin{array}{l}\text { Valor } \\
\text { máximo }\end{array}$ \\
\hline $\begin{array}{l}\text { Dopamina en orina } 24 \mathrm{~h}(<500 \\
\mathrm{mcg} / 24 \mathrm{~h})\end{array}$ & $\begin{array}{l}\text { Cromatografía líquida de alta } \\
\text { resolución }\end{array}$ & 374 & 374 & 224 & 524 \\
\hline $\begin{array}{l}\text { Metanefrinas totales en orina } \\
24 \mathrm{~h}(<900 \mathrm{mcg} / 24 \mathrm{~h})\end{array}$ & $\begin{array}{l}\text { Cromatografía líquida de alta } \\
\text { resolución }\end{array}$ & 6411 & 4127 & 77 & 19051 \\
\hline Epinefrina plasma $(0-100 \mathrm{pg} / \mathrm{ml})$ & Inmunoensayo enzimático & 2321 & 2321 & $<1$ & 3835 \\
\hline $\begin{array}{l}\text { Metanefrina fraccionada en orina } \\
24 \mathrm{~h}(<300 \mathrm{mcg} / 24 \mathrm{~h})\end{array}$ & $\begin{array}{l}\text { Cromatografía líquida de alta } \\
\text { resolución }\end{array}$ & 1611 & 606 & 92 & 6509 \\
\hline $\begin{array}{l}\text { Metanefrinas en plasma } \\
(0,05-0,16 \mathrm{pg} / \mathrm{ml})\end{array}$ & $\begin{array}{l}\text { Cromatografía líquida de alta } \\
\text { resolución }\end{array}$ & 1865 & 1865 & 1865 & 1865 \\
\hline $\begin{array}{l}\text { Norepinefrina en orina } 24 \mathrm{~h} \\
(<80 \mathrm{mcg} / 24 \mathrm{~h})\end{array}$ & $\begin{array}{l}\text { Cromatografía líquida de alta } \\
\text { resolución }\end{array}$ & 1008 & 373 & 36,4 & 2617 \\
\hline $\begin{array}{l}\text { Norepinefrina en plasma } \\
(0-600 \mathrm{pg} / \mathrm{ml})\end{array}$ & Inmunoensayo enzimático & 1832 & 1832 & $<1$ & 29559 \\
\hline $\begin{array}{l}\text { Normetanefrina en orina } 24 \mathrm{~h} \\
(<600 \mathrm{mcg} / 24 \mathrm{~h})\end{array}$ & $\begin{array}{l}\text { Cromatografía líquida de alta } \\
\text { resolución }\end{array}$ & 8295 & 8537 & 512 & 13086 \\
\hline $\begin{array}{l}\text { Ácido vanil mandélico } \mathrm{mg} / 24 \mathrm{~h} \\
\text { (2-14 } \mathrm{mg} / 24 \mathrm{~h})\end{array}$ & Colorimetría & 545 & 11 & 5,4 & 2692 \\
\hline $\begin{array}{l}\text { Epinefrina en orina } 24 \mathrm{~h}(<100 \\
\mathrm{mcg} / 24 \mathrm{~h})\end{array}$ & $\begin{array}{l}\text { Cromatografía líquida de alta } \\
\text { resolución }\end{array}$ & 1321 & 721 & 6,5 & 3835 \\
\hline
\end{tabular}

Análisis del perfil bioquímico de los pacientes con base en la media, mediana y los valores mínimo y máximo presentados en cada una de las pruebas realizadas.

Tabla 3. Manifestaciones clínicas

\begin{tabular}{l|c|c}
\hline \multicolumn{1}{c|}{ Manifestaciones clínicas } & N & \% \\
\hline Hipertensión arterial & 9 & 81 \\
\hline Cefalea & 3 & 27 \\
\hline Dolor precordial & 3 & 27 \\
\hline Dolor abdominal & 3 & 27 \\
\hline Hematuria & 1 & 9 \\
\hline Disnea & 1 & 9 \\
\hline Palpitaciones & 3 & 27 \\
\hline Frialdad y palidez & 2 & 18 \\
\hline Miopatía & 1 & 9 \\
\hline
\end{tabular}

Proporción de los signos y síntomas presentados en los pacientes del estudio.

control con menos dosis de medicamentos que en el prequirúrgico. Ningún paciente falleció durante el posquirúrgico. La evolución posterior de dos pacientes al egreso es desconocida pues no hay reportes en la historia clínica, y porque fueron remitidos a otras instituciones. Entre los demás pacientes, sólo el paciente con feocromocitoma maligno tuvo una respuesta desfavorable a pesar de manejo quirúrgico y quimioterapia y en el momento se encuentra en cuidado paliativo.

\section{Discusión}

El feocromocitoma es una rara neoplasia endocrina que secreta catecolaminas y proviene de las células cromafines de la médula adrenal. Lleva a complicaciones producto del exceso de catecolaminas $^{(1)}$. En nuestra revisión, en un periodo de 10 años se encontraron 11 pacientes con diagnóstico de feocromocitoma confirmado por patología, dentro de los cuales $45 \%$ eran hombres en la quinta década de la vida en su mayoría con hallazgos por imagen, a los que luego se les realizó perfil bioquímico e histopatológico. Todos los casos se trataron de lesiones unilaterales, una sola paciente tenía paraganglioma extraadrenal y uno con feocromocitoma maligno extraadrenal. El promedio del tamaño de las lesiones fue de $4,7 \mathrm{~cm}$. En cuanto al perfil bioquímico de los pacientes, las pruebas realizadas fueron metanefrinas totales calculadas en plasma, medición por colorimetría de ácido vanil mandélico en orina de 24 horas, y metanefrinas totales en orina de 24 horas medidas por cromatografía, en los casos que no se tomaron, no existía la sospecha previa de este diagnóstico, lo que no permitió confirmar el diagnóstico por este método previo a la cirugía ni tampoco se hizo preparación con manejo farmacológico antihipertensivo; sin embargo, no se presentaron complicaciones intra o perioperatorias por crisis hipertensiva. Sólo se presentó una complicación hemorrágica que requirió soporte transfusional por infiltración de la lesión hacia el lóbulo hepático derecho, que fue controlada rápidamente.

Las manifestaciones clínicas son similares a lo reportado en la literatura, siendo HTA, cefalea y palpitaciones las más comunes $^{(4)}$. En nuestro trabajo, el $81 \%$ presentaban hipertensión arterial al momento del diagnóstico y después de la resección del tumor el 67\% lograron cifras tensionales normales; los demás pacientes se controlaron con menos medicamentos que los utilizados en el prequirúrgico. En la literatura se reportan tasas de curación de HTA hasta del 95\%, esto reafir- 
ma la importancia de la identificación y tratamiento de esta patología como una causa potencialmente curable de $\mathrm{HTA}^{(5,6)}$. En nuestra serie no encontramos un patrón clínico, pero es de destacar que el $18 \%$ eran totalmente asintomáticos, siendo el diagnóstico incidental.

Aunque alguna vez la resección laparoscópica fue contraindicación, recientemente con la mejoría de las técnicas quirúrgicas se ha vuelto la estrategia de elección ${ }^{(7)}$. En nuestra serie, el $55 \%$ de los pacientes se sometieron a resección tumoral vía laparoscópica; es de resaltar la resección por vía laparoscópica de un tumor de 7,0 cm, que hasta hoy sería una contraindicación relativa por el tamaño tumoral y alta probabilidad de complicaciones. En nuestro caso la extracción fue exitosa, siendo importante contar con un grupo quirúrgico experimentado.

La preparación antes de la cirugía es fundamental, el manejo estándar es el uso de alfa y betabloqueadores con el ánimo de evitar la crisis hipertensiva antes y durante la cirugía. En nuestra experiencia, sólo 45\% recibieron alfa y betabloqueador, encontrando que un $27 \%$ presentaron hipertensión a la manipulación de tumor a pesar de recibir alfa y betabloqueo previo. Se deben destacar los casos en los que no se usó alfabloqueador en vista de no presentar síntomas sugestivos de descarga adrenérgica o hipertensión arterial; evidencia aun no clara en la literatura, debido a que el alfabloqueo en pacientes normotensos es reportado en algunas series con una mayor proporción de requerimiento de soporte vasoactivo ${ }^{(8)}$.

Lo anterior nos introduce en una de las complicaciones posoperatorias más temidas, sin duda la hipotensión, que ocurre inmediatamente posterior a la resección quirúrgica y requiere el manejo de vasopresores y de expansión agresiva del volumen plasmático ${ }^{(9)}$, esta complicación se encontró en el $45 \%$ de los pacientes de esta serie.

Se han descrito diferentes predictores de hipotensión como: tamaño tumoral $>60 \mathrm{~mm}$, niveles de epinefrina en orina $>200 \mathrm{mcg} / 24 \mathrm{~h}$, norepinefrina $>600 \mathrm{mcg} / 24 \mathrm{~h}$ y el tipo de medicación previa o alfabloqueo usado, siendo la fenoxibenzamina asociada con mayores tasas de hipotensión ${ }^{(10,11)}$. En el análisis de los casos el promedio en el tamaño de las lesiones fue de $47 \mathrm{~mm}$, con un promedio de epinefrina en orina de $1.321 \mathrm{mcg}$ en $24 \mathrm{~h}$ y de norepinefrina en $1.008 \mathrm{mcg}$ en $24 \mathrm{~h}$. De los seis casos en quienes se documentó hipotensión posquirúrgica, cinco habían sido premedicados con betabloqueador y/o prazosina, ninguno recibió fenoxibenzamina.

La prevalencia de feocromocitomas malignos en la literatura ha sido reportada del $5-26 \%^{(7,12)}$. No hay marcadores histológicos o bioquímicos que distingan tumores benignos de malignos. El diagnóstico de malignidad requiere la evidencia de metástasis a un sitio donde las células cromafines no existen. Persisten aún controversias en el diagnóstico, tratamiento y pronóstico, siendo la sobrevida menor al 50\% sin tratamiento ${ }^{(12,13)}$. Nosotros a la fecha sólo reportamos un paciente con metástasis óseas al momento del diagnóstico y fue tratado con quimioterapia con persistencia de hipertensión y niveles de metanefrinas y catecolaminas en orina elevadas, quien completa 36 meses de sobrevida con aceptable control de su presión arterial recibiendo metoprolol y losartán y manejo paliativo con radioterapia de metástasis óseas.

En cuanto a reportes de mortalidad en el seguimiento en nuestra población no hubo ningún caso de muerte durante o posterior a la intervención quirúrgica; sin embargo, la evolución de dos pacientes posterior al egreso es desconocida pues no hay reportes en la historia clínica, y porque fueron remitidos a otras instituciones.

\section{Conclusión}

Los feocromocitomas, si bien son tumores poco frecuentes, representan un alto riesgo de morbilidad y mortalidad; es importante sensibilizarnos con su diagnóstico de forma oportuna e implementar un manejo adecuado para impactar positivamente en los desenlaces. Las características de nuestra población son similares a lo reportado en la literatura, con baja frecuencia de complicaciones y adecuada respuesta al tratamiento.

\section{Referencias}

1. Lenders J, Eisenhofer G, Mannelli M, Pacak K. Phaeochromocytoma. Lancet [Internet]. 2005; Available from: http://linkinghub.elsevier.com/retrieve/ pii/S0140673605671395\npapers2://publication/uuid/05CB9BF0B7B4-4459-A95F-EB86E48D8365.

2. Manger WM, Gifford RW. Pheochromocytoma. J Clin Hypertens [Internet]. 2002;4(1):62-72. Available from: http://www.ncbi.nlm.nih.gov/ entrez/query.fcgi? $\mathrm{cmd}=$ Retrieve $\& \mathrm{db}=$ PubMed \&dopt=Citation\&list uids $=11821644$.

3. D’Achiardi R, Torres H, Guzmán J, Ordoñez JD. Feocromocitoma: presentación de seis casos. Acta Médica Colomb. 1980;5(3):453-63.

4. Shlomo Nelmed, Keneth Polonsky, P. Reed Larsen HMK. Williams Textbook of Endocrinology. 12th ed. Saunders E, editor. 2011. 548-549.

5. Castilho LN, Simoes F a, Santos a M, Rodrigues TM, Dos Santos Jr. C a. Pheochromocytoma: A long-term follow-up of 24 patients undergoing laparoscopic adrenalectomy. Int Braz J Urol [Internet]. 2009;35:24-31. Available from: http://www.embase.com/search/results?subaction=viewrecord\&fr om=export\&id=L354826797.

6. Plouin PF, Chatellier G, Fofol I, Corvol P. Tumor recurrence and hypertension persistence after successful pheochromocytoma operation. Hypertension [Internet]. 1997;29(5):1133-9. Available from: http://www.ncbi.nlm. nih.gov/pubmed/9149678\nhttp://hyper.ahajournals.org.ezp-prod1.hul. harvard.edu/cgi/content/full/29/5/1133.

7. Edstr??m Elder E, Skog AHH, H????g A, Hamberger B. The management of benign and malignant pheochromocytoma and abdominal paraganglioma. Eur J Surg Oncol. 2003;29(3):278-83.

8. Shao Y, Chen R, Shen Z, Teng Y, Huang P, Rui W, et al. Preoperative alpha blockade for normotensive pheochromocytoma: is it necessary? J Hypertens [Internet]. 2011;29(12):2429-32. Available from: http://www.ncbi. nlm.nih.gov/pubmed/22025238.

9. Kiernan CM, Du L, Chen X, Broome JT, Shi C, Peters MF, et al. Predictors of hemodynamic instability during surgery for pheochromocytoma. Ann Surg Oncol [Internet]. 2014;21(12):3865-71. Available from: http://www.ncbi. nlm.nih.gov/pubmed/24939623.

10. Weingarten TN, Cata JP, O'Hara JF, Prybilla DJ, Pike TL, Thompson GB, et al Comparison of two preoperative medical management strategies for laparoscopic resection of pheochromocytoma. Urology. 2010;76(2):508.

11. Namekawa T1, Utsumi T2 KK. Clinical predictors of prolonged postresection hypotension after laparoscopic adrenalectomy for pheochromocytoma. Surgery. 2016;159(3):763-70.

12. Eisenhofer G, Bornstein SR, Brouwers FM, Cheung NK V, Dahia PL, De Krijger RR, et al. Malignant pheochromocytoma: Current status and initiatives for future progress. Endocrine-Related Cancer. 2004. p. 423-36.

13. Huang KH, Chung SD, Chen SC, Chueh SC, Pu YS, Lai MK, et al. Clinical and pathological data of 10 malignant pheochromocytomas: Long-term follow up in a single institute. Int J Urol. 2007;14(3):181-5. 